\title{
Review of: "A fresh understanding of the Mpemba effect"
}

\author{
Changqing Sun ${ }^{1}$ \\ 1 Nanyang Technological University
}

Potential competing interests: The author(s) declared that no potential competing interests exist.

The work [1] described a fresh understanding of the Mpemba Effect in terms of the generic nonequilibrium statistic approach of Lu and Raz [2] and a recent experimental using feedback laser tweezers by Kumar and Bechhoefer [3]. The theory for small systems predicted the existence of an inverse Markovian Mpemba effect (IMME) that occurs during heating: when two systems are heated by the same hot bath, the initially colder system take a shorter time to heat. This IMME effect was demonstrated on both the three-state system and the antiferromagnetic Ising chain. The experiment [3] showed that a single micrometer-sized hot colloidal particle immersed in water cools faster. This is realized by controlling a water-like, asymmetrical two-dip potential: one dip corresponds to the "supercool" of water at subzero temperatures, and a lower dip to water freezes. By adjusting the potential shape yields conditions to ensure the speed-up in the particle cooling.

I would like to recommend that water is a more complex system [4, 5]. Further caution may be needed when applying the theory [2] to water, as the microscopic mechanism may differ: Water skin possesses higher thermal diffusivity and lower specific and the manner of hydrogen bond (O:H-O) energy exchange is different [6].

Figure 1 shows the reproduction of the Mpemba characteristics using the Fourier Fluid thermodynamics. It suggests that the Mpemba effect integrates the $\mathrm{O}: \mathrm{H}-\mathrm{O}$ bond energy "heating absorption, cooling emission, crossing skin-bulk interface diffusion, and source-drain interface dissipation" dynamics of liquid water [7]. One needs to consider the "source-path-drain" circulating as a unary in dealing with this system. Quantitative reproduction of the thermal decay and the skin-bulk temperature difference revealed the factors pertained to the rate of water-ice thermodynamic transition:

1. O:H-O bond memorability. Mpemba effect could only happen to systems with the coupled $\mathrm{O}: \mathrm{H}-\mathrm{O}$ bond, capable of absorbing energy at heating by $\mathrm{H}-\mathrm{O}$ contraction and emitting energy at its inverse. Presence of the lone pairs and the $\mathrm{O}-\mathrm{O}$ repulsion ensured the $\mathrm{O}: \mathrm{H}-\mathrm{O}$ bond memorability.

2. Skin supersolidity. Only the thermal diffusion, $a=k /\left(r C_{p}\right)$, and convection are involved in the Fourier fluid thermodynamics. $\mathrm{O}: \mathrm{H}-\mathrm{O}$ bond elongation by the skin molecular undercoordination lowers the skin 
mass density $r=0.75 \mathrm{~g} / \mathrm{cm}^{3}$ and specific heat to promote the thermal diffusivity, easing heat outward flow.

3. Non-adiabatic heat dissipation. Non-adiabatic source-drain interface is necessary to ensure the Mpemba effect extrinsically. Any measures hindering heat dissipation will prevent this effect from being happen.

4. Critically intrinsic conditions. The Mpemba effect should happen only to systems with involvement of strong correlation of the intermolecular bonding and intramolecular nonbonding interactions and the skin higher thermal diffusivity dominated by the lower skin mass density. Mpemba effect happens unlikely to regular substance because the skin bond contraction lowers the thermal diffusivity, and the cooling bond contraction absorbs energy.

5. The supercooling cools slowly. Induced by electrification, molecular undercoordination, or pressure lower than the ambient, the $\mathrm{H}-\mathrm{O}$ contraction derived the supercooling, and the shorter $\mathrm{H}-\mathrm{O}$ bond is hardly deformed.

Figure 1 illustrates (a) the potential paths and (b) the initial temperature dependence of the liner velocity of the $\mathrm{O}: \mathrm{H}-\mathrm{O}$ bond during thermal relaxation [4]. Heating stores energy in water by stretching the $\mathrm{O}: \mathrm{H}$ nonbond and simultaneously compressing the $\mathrm{H}-\mathrm{O}$ bond (red line linked spheres in Figure $1 \mathrm{a}$ are in the hot state) by the coupling interactions. Cooling reverses (blue line linked spheres) this process, analogous to suddenly releasing a coupled, highly deformed bungee pair, one of which is stretched and the other compressed. This process emits energy at a rate that depends on the deformation history of the bungee pair (i.e., how much they were stretched or compressed) [8].

The O:H-O bond exhibits memory with thermal momentum during cooling. The linear velocity of the $\mathrm{H}-\mathrm{O}$ bond $\mathrm{Dd}_{\mathrm{H}} / \mathrm{Dt}$ in Figure $\mathrm{Ib}$ is the product of slopes for the known temperature dependence of $\mathrm{H}-\mathrm{O}$ length $d_{H}(q)$ and the measured initial temperature and time dependence of water temperature $q\left(q_{i}, t\right)$ shown in Figure $1 \mathrm{c}$ as inset. Because of the Coulomb coupling of the $\mathrm{O}: \mathrm{H}$ and $\mathrm{H}-\mathrm{O}$, the velocity $\mathrm{Dd} \mathrm{d}_{\mathrm{x}} / \mathrm{Dt}$ and energy rate $\mathrm{DE}_{\mathrm{x}} / \mathrm{Dt}(\mathrm{x}=\mathrm{L}$ for $\mathrm{O}: \mathrm{H}$ and $\mathrm{x}=\mathrm{H}$ for $\mathrm{H}-\mathrm{O}$ ) can be readily derived but here we show the representative only for simplicity. Figure $\mathrm{lb}$ indicates that the initially shorter $\mathrm{H}-\mathrm{O}$ bond at higher temperature remains highly active compared to its behavior otherwise when they meet on the way to freezing [8].

Conversely, molecular undercoordination (with fewer than four neighbor as in the bulk) has the same effect of heating on O:H-O bond relaxation [9] (https://doi.org/10.32388/FO6KAP). H-O bond contracts and O:H expands, which shrinks molecular size and expands their separations with an association of polarization [10]. Water skin forms such a supersolid phase that is elastic, hydrophobic, ice like, and less dense $\left(0.75 \mathrm{gcm}^{-3}\right)$. The lower mass density raises the thermal diffusivity and favors outward heat flow from the liquid. 
Figure 1c and d show the theoretical duplication of measured (insets) Mpemba paradox [8]. The Mpemba paradox is characterized by [11]: (i) hot water freezes faster than cold water under the same conditions; (ii) the liquid temperature $q$ drops exponentially with cooling time (t); (iii) water skin is warmer than sites inside the liquid and the skin of hotter water is even warmer.

It is necessary to emphasize that the Mpemba effect happens only in circumstances where the water temperature drops sharply from $q_{i}$ to $q_{f}$ at the source-drain interface. Larger liquid volume may prevent this effect by heat-dissipation hindering. Any spatial temperature decay between the source and the drain could prevent the Mpemba effect. Conducting experiments under identical conditions is necessary to minimize artifacts such as radiation, source/drain volume ratio, exposure area, container material, etc. Therefore, conditions for the Mpemba effect are indeed very critical, which explains why it is not frequently observed.
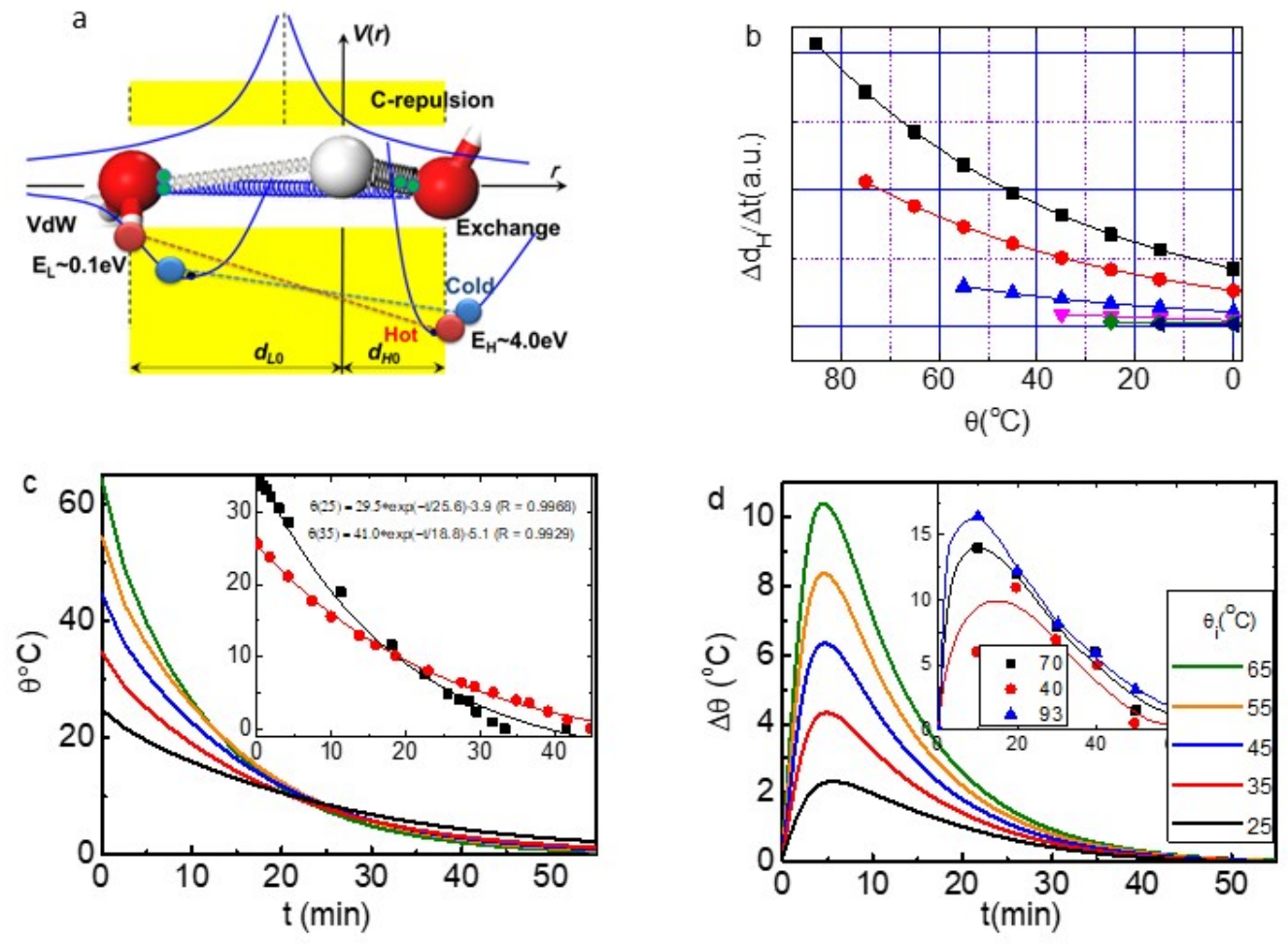

Figure 1. (a) O:H-O bond asymmetric, short-range potential paths, being analogous to springs and (b) O:H-O bond memory effect - initial temperature dependence of the $\mathrm{H}-\mathrm{O}$ bond relaxation velocity at cooling. Numerical duplication of the measured (insets) initial temperature dependence of the $(c) q\left(q_{i}, t\right)$ curves [11] and $(d)$ the skin-bulk temperature difference $q\left(q_{i}, t\right)$ of water at cooling [4]. The $d_{\mathrm{HO}}$ and $d_{\mathrm{LO}}$ in (a) are the respective references at $4^{\circ} \mathrm{C}$. Potentials include the O:H nonbond interaction (vdW 
of $E_{\mathrm{L}} \sim 0.1 \mathrm{eV}$ bond energy; left-hand side), the $\mathrm{H}-\mathrm{O}$ bond exchange interaction $\left(E_{\mathrm{H}} \sim 4.0 \mathrm{eV}\right.$; right-hand side), and the Coulomb repulsion between electron pairs (paired green dots) on adjacent oxygen atoms. These interactions dislocate $\mathrm{O}$ atoms in the same direction by different amounts with respect to the $\mathrm{H}^{+}$origin under excitation. The relaxation proceeds along the O:H-O bond potentials from hotter (red line linked spheres, labelled 'hot') to colder state (blue line linked spheres, labelled 'cold').

Therefore, O:H-O bond memory and water-skin supersolidity determines intrinsically and the skin-drain interface nonadiabatic dissipation extrinsically the heat 'emission-conduction-dissipation' dynamics of the Mpemba paradox in the 'source-path-drain' cycle system.

1. Bechhoefer, J., A. Kumar, and R. Chétrite, A fresh understanding of the Mpemba effect. Nature Reviews Physics, 2021: p. 1-2.

2. Lu, Z. and O. Raz, Nonequilibrium thermodynamics of the Markovian Mpemba effect and its inverse. Proceedings of the National Academy of Sciences, 2017. 114(20): p. 5083-5088.

3. Kumar, A. and J. Bechhoefer, Exponentially faster cooling in a colloidal system. Nature, 2020. 584(7819): p. 64-68.

4. Sun, C.Q., X. Zhang, X. Fu, W. Zheng, J.-I. Kuo, Y. Zhou, Z. Shen, and J. Zhou, Density and phononstiffness anomalies of water and ice in the full temperature range. Journal of Physical Chemistry Letters, 2013. 4: p. 3238-3244.

5. Sun, C.Q., X. Zhang, J. Zhou, Y. Huang, Y. Zhou, and W. Zheng, Density, Elasticity, and Stability Anomalies of Water Molecules with Fewer than Four Neighbors. Journal of Physical Chemistry Letters, 2013. 4: p. $2565-2570$.

6. Demming, A., Experiments pin down conditions that make hot water freeze before cold. Physics World, 2020. 05 Aug 2020.

7. Zhang, X., Y. Huang, Z. Ma, Y. Zhou, J. Zhou, W. Zheng, Q. Jiang, and C.Q. Sun, Hydrogen-bond memory and water-skin supersolidity resolving the Mpemba paradox. Physical Chemistry Chemical Physics, 2014. 16(42): p. 22995-23002.

8. Zhang, X., Y. Huang, Z. Ma, Y. Zhou, W. Zheng, Q. Jiang, and C.Q. Sun, Hydrogen-bond memory and water-skin supersolidity resolving Mpemba paradox. PCCP, 2014: p. DOI: 10.1039/C4CP03669G.

9. Zhang, X., Y. HUang, Z. Ma, J. Zhou, Y. Zhou, W. Zheng, and C.Q. Sun, A common superslid skin covering both water and ice. PCCP, 2014: p. DOI: 10.1039/C4CP02516D.

10. Sun, C.Q., X. Zhang, J. Zhou, Y. Huang, Y. Zhou, and W. Zheng, Density, Elasticity, and Stability Anomalies of Water Molecules with Fewer than Four Neighbors. The Journal of Physical Chemistry Letters, 2013. 4: p. 2565-2570.

11. Bregović, N., Mpemba effect from a viewpoint of an experimental physical chemist. http://www.rsc.org/images/nikola-bregovic-entry_tcm18-225169.pdf, 2012. 
